\title{
Contenido de metales pesados y composición química de los cementos hidráulicos de uso general comercializados en Costa Rica
}

\section{Content of Heavy Metals and Chemical Composition of the Hydraulic Cement Marketed in Costa Rica}

\author{
Jimmy Venegas Padilla \\ Investigador del Departamento de Metrología en Química, LACOMET, MEIC. \\ jvenegas@lacomet.go.cr \\ Bryan Calderón Jiménez* \\ Investigador del Departamento de Metrología en Química, LACOMET, MEIC. \\ bcalderon@lacoment.go.cr. \\ José Pablo Sibaja Brenes \\ Académico, Escuela de Química, Universidad Nacional, Costa Rica. \\ Jorge Salazar Delgado \\ Químico. Investigador. Unidad de Materiales y Pavimentos, Lanamme, UCR, Costa Rica \\ Ellen Rodríguez Castro \\ Investigadora, Laboratorio de Ligantes Asfálticos, Lanamme, UCR, Costa Rica
}

Recibido: 09 de noviembre 2016

Aceptado: 23 de febrero 2017

\section{Resumen}

El presente estudio tuvo como objetivo determinar el contenido de los metales pesados, específicamente el contenido de plomo $(\mathrm{Pb})$, cromo $(\mathrm{Cr})$ y mercurio $(\mathrm{Hg})$, y la composición química $\left(\mathrm{CaO}, \mathrm{SiO}_{2}, \mathrm{Al}_{2} \mathrm{O}_{3}, \mathrm{Fe}_{2} \mathrm{O}_{3}\right.$ entre otros) del cemento hidráulico de uso general comercializado en Costa Rica. Los parámetros físicos de densidad y finura confirmaron que los cementos analizados presentaron suficiente homogeneidad para evaluar el contenido de los constituyentes químicos y de los metales pesados. Los constituyentes químicos se determinaron mediante Fluorescencia de Rayos-X (XRF). Los cementos presentaron fracciones en masa en el ámbito de $\left(61,22\right.$ - 63,12) \% de $\mathrm{CaO},\left(18,10\right.$ - 26,14) \% de $\mathrm{SiO}_{2},(3,70-6,05) \%, \mathrm{Al}_{2} \mathrm{O}_{3},(2,57-3,36)$ $\%$ de $\mathrm{Fe}_{2} \mathrm{O}_{3}$ y $(0,60-4,09) \%$ de $\mathrm{MgO}$. Otros compuestos minoritarios tales como $\mathrm{MgO}, \mathrm{TiO}_{2}$, $\mathrm{K}_{2} \mathrm{O}, \mathrm{P}_{2} \mathrm{O}_{5}, \mathrm{Mn}_{2} \mathrm{O}_{3}$ y Na $\mathrm{Na}_{2} \mathrm{O}$ se encontraron en fracciones, promedio en masa, inferiores al 1 $\%$. Además, por medio de los resultados obtenidos en la prueba de ignición y asumiendo una descarbonatación total de la caliza se pudo estimar de manera indirecta el contenido de $\mathrm{CaCO}_{3}$ y $\mathrm{CaO}$ aportados por la materia prima. El contenido de metales pesados se determinó por medio de las técnicas de Espectroscopia de Absorción Atómica con Llama (FAAS), Espectroscopia de 
Absorción Atómica con Atomización Electrotérmica (ETAAS) y Espectroscopia de Absorción Atómica con Generación de Vapor Frío (CVAAS). El estudio demostró que el Pb se encontró en concentración variable desde los $(2,45 \pm 0,72) \mathrm{mg} \mathrm{kg}^{-1}$ hasta los $(8,95 \pm 1,34) \mathrm{mg} \mathrm{kg}^{-1}$, el Cr presentó concentraciones de hasta $(10,69 \pm 0,92) \mathrm{mg} \mathrm{kg}^{-1}$. Se determinó que el $\mathrm{Hg}$ se encontraba en concentraciones inferiores a los $(0,141 \pm 0,021) \mathrm{mg} \mathrm{kg}^{-1}$. En términos generales, los cementos hidráulicos de uso general comercializados en Costa Rica presentaron una composición química mineralógica similar a varios tipos de cementos comercializados en Alemania. Los resultados del contenido de los metales pesados expuestos en este estudio brindarán información sustancial para los futuros estudios en las áreas de toxicológica, ecotoxicológica, normalización y en la reglamentación nacional.

\title{
Palabras Clave:
}

Cemento Hidráulico, Composición química, Metales pesados, Plomo, Cromo, Mercurio

\begin{abstract}
The present study aims to quantify the concentration of heavy metals, specifically lead $(\mathrm{Pb})$, chromium $(\mathrm{Cr})$, and mercury $(\mathrm{Hg})$, and also the chemical composition $\left(\mathrm{CaO}, \mathrm{SiO}_{2}, \mathrm{Al}_{2} \mathrm{O}_{3}, \mathrm{Fe}_{2} \mathrm{O}_{3}\right.$ among others) of the hydraulic cement marketed in Costa Rica. The physical parameters of density and fineness confirmed the homogeneity of the samples to determinate accurately the content of the major components and heavy metals in the cements. The mineralogical constitution was determined by X-ray Fluorescence (XRF). Specifically, the cements showed a mass fraction in the range of $(61.22-63.12) \%$ of $\mathrm{CaO},(18.10-26.14) \%$ of $\mathrm{SiO}_{2},(3.70-6.05) \%$ of $\mathrm{Al}_{2} \mathrm{O}_{3},(2.57-$ 3.36) $\% \mathrm{Fe}_{2} \mathrm{O}_{3}$ and $(0.60-4.09) \%$ de $\mathrm{MgO}$. Other components such as $\mathrm{MgO}, \mathrm{TiO}_{2}, \mathrm{~K}_{2} \mathrm{O}, \mathrm{P}_{2} \mathrm{O}_{5}$, $\mathrm{Na}_{2} \mathrm{O}$ and $\mathrm{Mn}_{2} \mathrm{O}_{3}$ were found on an average mass fraction lower than $1 \%$. Moreover, using the ignition test results and assuming a complete decomposition of the limestone, it was possible to estimate (indirectly) the content of $\mathrm{CaCO}_{3}$ and $\mathrm{CaO}$ given by the raw materials. The metal content of the heavy metals was determined using Flame Atomic Absorption Spectroscopy (FAAS), Electrothermal Atomic Absorption Spectroscopy (ETAAS), and Cold Vapor Atomic Absorption Spectroscopy (CVAAS). The analysis demonstrated that the $\mathrm{Pb}$ in cements is present in different concentrations ranging the $(2.45 \pm 0.72) \mathrm{mg} \mathrm{kg}^{-1}$ to the $(8.95 \pm 1.34) \mathrm{mg} \mathrm{kg}^{-1}$. Chromium $(\mathrm{Cr})$ was presented in higher concentrations of $(10.69 \pm 0.92) \mathrm{mg} \mathrm{kg}^{-1}$. The $\mathrm{Hg}$ concentration was below $0.141 \pm 0.021 \mathrm{mg} \mathrm{kg}^{-1}$. In general terms, the hydraulic cements marketed and used in Costa Rica have a suitable chemical composition compared with some cements marketed in Germany. The results of the content of heavy metals presented in this study provide significant information for future studies in the area of toxicology, ecotoxicology, standardization and national regulation.
\end{abstract}

Keywords: Hydraulic Cement, Chemical Composition, Heavy Metals, Lead, Chromium, Mercury 


\section{INTRODUCCIÓN}

Actualmente, la industria del cemento ha promovido la co-incineración o el co-procesamiento como una actividad que permite el uso de combustibles alternativos en los procesos de calcinación, así como materias primas alternativas para la manufactura del cemento. El objetivo de esta actividad en las industrias es sustituir los combustibles fósiles y/o las materias primas naturales por los residuos que permitan reducir los costos de producción y la huella medioambiental, sin disminuir la calidad del producto final (1). La manufacturación del cemento requiere de un consumo energético muy elevado, debido a las altas temperaturas que son requeridas en el proceso. Varios estudios $(2,3)$ han reportado que los subproductos del cloruro, las cenizas y los residuos provenientes de la producción de los carbonatos, combinados con la piedra caliza, disminuyen la temperatura de calcinación para la producción del clinker, lo cual representa una ventaja energética en la industria. Residuos de neumáticos, plásticos, biomasa, tela, partes desmanteladas de los autos, llantas en desuso y lubricantes a base de petróleo, son otras fuentes de combustibles alternativos que la industria cementera utiliza para la producción del cemento $(2,3)$.

El cemento hidráulico en general se encuentra constituido por una gran variedad de elementos y compuestos, los cuales podrían clasificarse en tres categorías: A) constituyentes químicos mayoritarios, B) constituyentes químicos minoritarios y C) elementos traza. Los constituyentes químicos mayoritarios son principalmente óxidos de calcio $(\mathrm{Ca})$, silicio $(\mathrm{Si})$, hierro $(\mathrm{Fe})$ y aluminio $(\mathrm{Al})$. Estos compuestos están en porcentajes mayores al $5 \%$ en la matriz del cemento. Además, es importante recalcar que el Ca, el Si y el Al, son los elementos más importantes en la conformación química del cemento, ya que estos son los responsables de la formación de las fases $\left(\mathrm{C}_{3} \mathrm{~S}, \mathrm{C}_{2} \mathrm{~S}, \mathrm{C}_{3} \mathrm{~A}\right.$ y $\left.\mathrm{C}_{4} \mathrm{AF}\right)$ que le confieren las propiedades físicas y mecánicas al cemento $(2,4,5)$.

Los constituyentes químicos minoritarios se encuentran en el cemento en porcentajes del 1-5\% en forma de óxidos. En esta clasificación se encuentra el magnesio (Mg), el potasio $(\mathrm{K})$, el sodio $(\mathrm{Na})$ y el azufre $(\mathrm{S})$, entre otros. Por último, los elementos traza se encuentran en un porcentaje pequeño en comparación con los otros constituyentes, representando el $0,02 \%$ en masa o en una concentración menor o igual a los $100 \mathrm{mg}$ $\mathrm{kg}^{-1}(12)$.

En este contexto, los elementos traza pueden provenir de fuentes naturales y antropogénicas, siendo este último lo más crítico, ya que las materias primas y los combustibles utilizados para la manufacturación del cemento aportan de forma inherente metales pesados a la constitución del cemento $(2,7,9)$. Lo anterior se debe a que los metales pesados y los metales en general, pueden ser introducidos al cemento por medio de los procesos de adsorción, incorporación química (acomplejamiento superficial, precipitación, co-precipitación) y microemcapsulación (8). Por otra parte, los materiales alternativos y auxiliares (escoria, esquisto bituminoso, cenizas volantes) utilizados en la producción del cemento son una fuente importante para incorporar elementos traza al cemento $(3,7)$, generando debate entre los científicos, los organismos reglamentarios y la sociedad, sobre cómo controlar y determinar el impacto real que genera este tipo de material en el ambiente y/o en la salud $(5,7,9)$. 
Algunos países de Europa han realizado investigaciones para estimar el contenido de los elementos traza incorporados por las materias primas y por los materiales alternativos en el cemento hidráulico, tales como el plomo $(\mathrm{Pb})$, el cromo $(\mathrm{Cr})$ y el mercurio $(\mathrm{Hg})(3,7,9,12)$. De igual manera, por medio de las agencias ambientales y de los institutos de investigación, en algunos países han establecido recomendaciones sobre la concentración que debe existir de estos elementos en las materias primas como el clinker, en el cemento y en los morteros a base de cemento $(6,12)$. Esto deja en manifiesto que el co-procesamiento de los residuos con alto contenido de elementos tóxicos se debe realizar de manera controlada, ya que hay una alta probabilidad de que los residuos aumenten la concentración habitual de los metales pesados en el cemento y, por consiguiente, se podría generar un impacto toxicológico y ambiental $(6,12)$.

Debido a lo anterior, es de suma importancia el control y el monitoreo de estos elementos en el cemento comercializado y distribuido hoy en día en Costa Rica. Por lo tanto, el presente estudio tuvo como objetivo realizar una caracterización para corroborar la homogeneidad y calidad de los cementos en estudio. Además, se realizó una caracterización química de los compuestos mayoritarios y minoritarios que constituyen los cementos hidráulicos evaluados. Finalmente, se evaluó el contenido del $\mathrm{Pb}$, el $\mathrm{Cr}$ y el $\mathrm{Hg}$ presente en los cementos comercializados en Costa Rica, con el objetivo primordial de establecer la concentración en que se encuentran estos elementos en los cementos de Costa Rica y, además, brindar una base científica para las futuras investigaciones.

\section{MATERIALES Y MÉTODOS}

Se seleccionaron cuatro puntos de distribución del cemento en el Gran Área Metropolitana (GAM), empleando un muestreo planificado en la zona de estudio. Se muestrearon solamente cementos de las marcas existentes en el mercado costarricense al momento del estudio. Los días de muestreo fueron seleccionados con al menos 30 días de diferencia. La secuencia de los comercios visitados se planeó en las direcciones de sur a norte y de este a oeste. El muestreo se realizó tomando como referencia la norma europea UNE-EN 196-7 (16), la cual especifica que los sacos muestreados fueron tomados al azar, asegurando que las muestras no presentasen problemas de humedad o algún daño en el empaque (16). Las muestras fueron homogenizadas sobre una lámina plástica inerte al cemento. Una vez homogenizada la muestra, fue cuarteada como se muestra en la Fig. 1. De cada cuarto de muestra se tomó una porción de sub-muestra hasta alcanzar una muestra representativa de aproximadamente $5 \mathrm{~kg}(16)$.

Las submuestras de cemento fueron colocadas en bolsas plásticas y claramente identificadas. Las submuestras se almacenaron en un cuarto de custodia con una temperatura inferior a $\operatorname{los} 30^{\circ} \mathrm{C}$ y con una relativa inferior al $40 \%$ (16).

El análisis de densidad del cemento hidráulico $(\rho)$ se realizó por el método estandarizado del matraz volumétrico de Le Châtelier descrito en el ASTM C188-09 (17), el cual se describe brevemente a continuación. Se utilizó un baño isotérmico a una temperatura de $(23,0 \pm 0,2){ }^{\circ} \mathrm{C}$, el cual fue controlado utilizando un termómetro digital tipo termorresistivo (RTD). La masa de cemento utilizada en el ensayo fue de aproximadamente 64,00 $\mathrm{g}\left(m_{c}\right)$. Las lecturas de volumen inicial y volumen final se tomaron cada 3 minutos hasta 


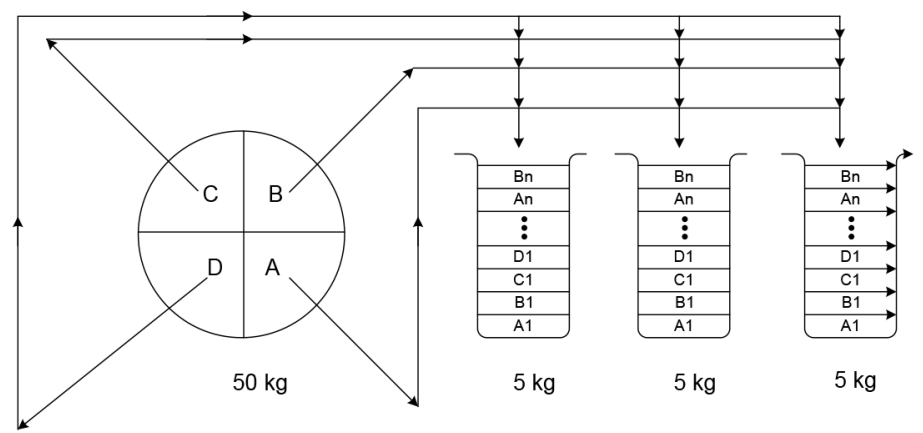

Figura 1. Esquema de cuarteo para la toma de muestra de cemento.

que se obtuvieron dos lecturas constantes para estimar el volumen desplazado $\left(V_{d}\right)$. El modelo del mesurando que describe el proceso de medición es el siguiente:

$$
\rho_{\left(\mathrm{g} / \mathrm{cm}^{3}\right)}=\frac{m_{c}(\mathrm{~g})}{V_{d}\left(\mathrm{~cm}^{3}\right)}
$$

El análisis de finura del cemento se realizó con base en el método húmedo con un tamiz No. 325 establecido por la norma ASTM C430-08 (18). Se utilizó un tamiz No. 325 (45 $\mu \mathrm{m})$ con las especificaciones que recomienda el ASTM E 11-16 y con su factor de corrección $(C)$ previamente determinado (33). A continuación, se describe brevemente el procedimiento empleado. Para cada replica se midió la masa, por pesada por diferencia, de aproximadamente $1,0000 \mathrm{~g}$ de cemento en una balanza analítica con una resolución de $0,1 \mathrm{mg}$ (18). Se colocó la muestra sobre el tamiz de $45 \mu \mathrm{m}$ y se humedeció por 1 min con una corriente de agua potable en forma de spray con una presión de $(69 \pm 4) \mathrm{kPa}$. El sólido retenido en el tamiz, se lavó con $50 \mathrm{ml}$ de agua desionizada y se secó en una estufa a $105^{\circ} \mathrm{C}$ por $2 \mathrm{~h}\left(R_{s}\right)$. Se determinó el residuo de muestra retenido en un tamiz de $45 \mu \mathrm{m}\left(R_{c}\right)$ por medio de la ecuación 2. Seguidamente, se procedió a realizar el cálculo porcentual de la finura del cemento hidráulico (F) por el tamiz de $45 \mu \mathrm{m}$ mediante el modelo matemático que se describe en la ecuación 3:

$$
\begin{gathered}
R_{c}(\%)=R_{s} \cdot(100+C) \\
F(\%)=100-R_{c}
\end{gathered}
$$

La determinación de la perdida por ignición se realizó de acuerdo con lo descrito en el apartado 18 de la norma ASTM C114-15 (19). A continuación, se describe brevemente el procedimiento empleado. Se midió una masa de 1,0000 g de cada muestra $\left(m_{i}\right)$ del cemento en un crisol de titanio, utilizando una balanza analítica con resolución de $0,1 \mathrm{mg}$. Posteriormente, se colocaron las muestras por 10 min en una mufla a 1000 
${ }^{\circ} \mathrm{C}$ (19). El crisol se atemperó en un desecador con $\mathrm{CaCl}_{2}$, y posteriormente se midió la masa final de la muestra $\left(m_{f}\right)$. Se procedió a determinar el porcentaje de la pérdida por ignición (P.I), empleando el modelo de mesurando descrito a continuación:

$$
\operatorname{P.I}(\%)=\frac{m_{i}-m_{f}}{m_{i}} \cdot 100
$$

Con los resultados reunidos en el apartado anterior, específicamente, la masa final de la muestra obtenida luego de la perdida por ignición $\left(m_{f}\right)$, se procedió a realizar el cálculo de la cantidad de fundente $\left(\mathrm{Li}_{2} \mathrm{~B}_{4} \mathrm{O}_{7}\right)$ y del cemento necesario para preparar las muestras para la determinación del contenido de los componentes mayoritarios y minoritarios por XRF (comúnmente denominada pastilla fundida) (19). La determinación se realizó utilizando un Espectrómetro de Fluorescencia de Rayos X por Energía Dispersiva (EDXRF, por sus siglas en inglés). El análisis se hizo con la colaboración de la industria nacional de cemento. Por políticas de confidencialidad no se detalla el nombre de la empresa ni se especifica a cabalidad el equipo, las condiciones de operación y los materiales utilizados en este apartado. Sin embargo, para mayor información sobre el método de análisis elemental por EDXRF, sus cálculos y otros aspectos técnicos se recomienda consultar las referencias $(40,41)$.

Para la determinación de metales pesados se realizó una digestión multietapas asistida por microondas. A continuación, se describe brevemente el proceso. Para más detalle sobre su desarrollo, ver lo descrito en la referencia (20). Se midió en una balanza de $0,1 \mathrm{mg}$ de exactitud una masa de $0,5 \mathrm{~g}$ de muestra de cemento y se transfirió a los recipientes de digestión. Se realizó un proceso de digestión multietapas, empleando $\mathrm{HNO}_{3}$, $\mathrm{HCl} / \mathrm{HF}$ y $\mathrm{H}_{3} \mathrm{BO}_{3}$. Una vez digerido el material, se almacenó en una incubadora a (20 $\pm 1)^{\circ} \mathrm{C}$ para su posterior medición.

La validación de los métodos y procedimientos utilizados para la determinación de $\mathrm{Pb}$, $\mathrm{Cr}$ y $\mathrm{Hg}$ en una matriz de cemento se detalla a profundidad por los autores en la referencia (20), por lo que a continuación se realizara una descripción breve del procedimiento utilizado. Se prepararon 5 patrones de calibración empleando un material de referencia de $\mathrm{Pb}$ trazable al NIST. Estos patrones se prepararon mediante diluciones gravimétricas. Las muestras fueron analizadas empleando un Espectrofotómetro de Absorción Atómica, Marca VARIAN, modelo AA240 FS, en modalidad de aspiración directa. Para las muestras que presentaron concentraciones de $\mathrm{Pb}$ por debajo de lo establecido en los límites de cuantificación del método por FAAS, se utilizó la técnica de Espectrofotometría de Absorción Atómica con Horno de Grafito (GFAAS) (20). El equipo empleado para este propósito fue un PerkinElmer, modelo PinAAcle 900T, con automuestreador marca PerkinElmer, modelo AS 900 y con corrección longitudinal de efecto Zeeman. El Cr se determinó empleando la técnica de FAAS y el mismo equipo que se utilizó para medir el elemento $\mathrm{Pb}$. Se prepararon 5 patrones de calibración empleando un material de referencia de $\mathrm{Cr}$ trazable al NIST. Estos patrones se prepararon mediante diluciones gravimétricas en diferentes concentraciones (20). Para la determinación de $\mathrm{Hg}$, se prepararon 5 patrones de calibración empleando un material de referencia de $\mathrm{Hg}$ trazable al NIST. El equipo PerkinElmer, FIAS 100 fue utilizado para generar la atomización 
en vapor frío para la determinación de $\mathrm{Hg}$. La cuantificación se realizó empleando el Espectrofotómetro PerkinElmer, modelo PinAAcle 900T con un automuestreador marca PerkinElmer, modelo S 10.

\section{RESULTADOS Y DISCUSIONES}

En la Fig. 2 se indican las localidades de los comercios donde se llevó a cabo el muestreo en el GAM. El muestreo se diseñó de tal manera que se pudiesen obtener muestras de los sectores Norte, Sur, Este y Oeste del GAM. Cada uno de los cementos muestreados fue identificado con una codificación única según el sistema de cómputo de entrega e ingreso de las muestras. Como se mencionó en el apartado anterior las muestras fueron conservadas en un cuarto custodia con condiciones de humedad y temperatura controladas, con la finalidad de conservar las propiedades físicas y químicas del material.

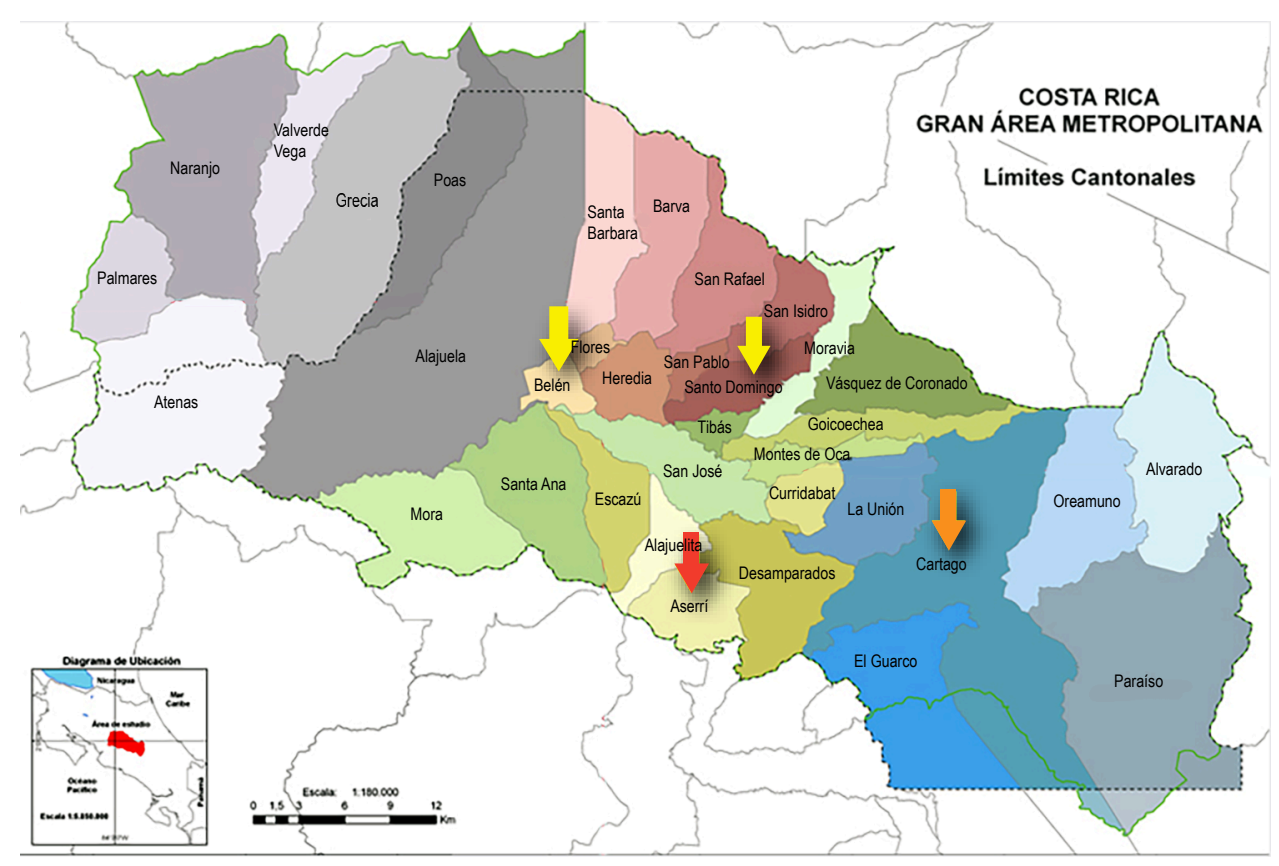

Figura 2. Puntos de muestreo del cemento en el GAM.

Disponible en: 201.194.102.38/cartografía/PRUGAM_Cartografia_Cantones.html

El análisis de densidad expone que los cementos muestreados presentaron valores que se distribuyen entre $(2,84 \pm 0,25) \mathrm{g} \mathrm{cm}^{-3}$ hasta $(2,98 \pm 0,22) \mathrm{g} \mathrm{cm}^{-3}$. Las pequeñas variaciones en estos resultados (ver Fig. 3.A) se deben primordialmente a las diferencias entre las tecnologías de triturado, clinkerizado y mezclado que cada empresa utilizó para la producción del cemento en Costa Rica. Por otra parte, el método para la determinación de la densidad presentó un buen desempeño en términos de su repetibilidad, cumpliendo con lo recomendado por la norma ASTM C188 (17). 
Los porcentajes promedio de la finura determinados en los cementos muestreados se detallan en la Fig. 3.B. Al igual que en la determinación de la densidad, se observaron diferencias pequeñas entre las finuras (utilizando tamiz de $45 \mu \mathrm{m}$ ) de los cementos muestreados. La mayor diferencia se observó al comparar la finura obtenida entre los cementos producidos por las distintas empresas. Estas diferencias son parte de las variaciones aleatorias de cada proceso productivo, las cuales se asocian directamente al tipo de materia prima y al nivel de eficiencia de los molinos de cada industria cementera (23, 24). Pese a lo anterior, los valores promedio de densidad y finura mostraron una alta homogeneidad en los cementos de cada empresa, siendo este un factor predominante en la determinación de los metales pesados en el cemento, ya que permitió corroborar que las muestras de cementos tenían la suficiente homogeneidad (en términos de tamaño de partícula y densidad) para realizar la determinación del contenido de $\mathrm{Cr}, \mathrm{Pb}$ y $\mathrm{Hg}$, sin que este factor tuviese un papel preponderante en la medición y su incertidumbre. Además, los valores de finura elevados favorecieron el proceso de digestión del cemento, lo cual es de gran importancia para una adecuada cuantificación de los elementos traza en los cementos $(20,21)$.

A nivel de residuos sólidos (ver Fig. 3.C), se observa que el contenido promedio de los residuos sólidos retenidos en el tamiz de $45 \mu \mathrm{m}$ fueron inferiores al $8 \%$, lo cual es un valor recomendado en la literatura (40). Lo anterior es de suma importancia para que los cementos hidráulicos presenten hidratación y, por consiguiente, el desarrollo de sus propiedades mecánicas de una forma rápida y eficiente $(23,25)$. Cabe resaltar que los porcentajes bajos de los residuos sólidos obtenidos en este estudio demuestran que los cementos analizados presentan un bajo contenido de aglomeraciones y agregaciones que afectarían la homogeneidad del cemento, generando una dispersión de los resultados en el análisis de los componentes mayoritarios y de los metales pesados, por consiguiente, produciendo, un impacto en la exactitud y la precisión de las mediciones.

En el tabla 1 se muestran los resultados de la determinación de la constitución química de los cementos hidráulicos comercializados en Costa Rica, empleando la técnica de XRF. Como se puede observar, los cementos analizados en este estudio presentaron porcentajes elevados de $\mathrm{CaO}, \mathrm{SiO}_{2}, \mathrm{Al}_{2} \mathrm{O}_{3}$ y $\mathrm{Fe}_{2} \mathrm{O}_{3}$, lo cual sugiere que en su proceso de formulación se utilizaron fuentes con alto contenido de este tipo de minerales tales como: caliza, clinker de cemento portland, escoria granulada de alto horno, materiales puzólanico y/o humo de silice. Este resultado es congruente con lo establecido en el antiguo reglamento técnico (27), el nuevo reglamento técnico de cemento (34) y la norma nacional de cemento (26), los cuales facultan la utilización de estos materiales para la producción de los cementos que se comercializan en Costa Rica. Debe recalcarse que los constituyentes químicos mayoritarios, $\mathrm{CaO}, \mathrm{SiO}_{2}, \mathrm{Al}_{2} \mathrm{O}_{3}$ y Fe $\mathrm{O}_{3}$, en el cemento son los responsables de brindar las propiedades mecánicas (específicamente resistencia a la compresión) del cemento hidráulico analizado. Además, es importante destacar que los componentes mayoritarios y minoritarios no son parámetros de calidad evaluados o establecidos en el antiguo reglamento técnico (27), el nuevo reglamento técnico de cemento (34) y la nueva norma nacional de cemento (26), generando que haya escasa información acerca de cómo se distribuyen estos componentes (mayoritarios y minoritarios) en los cementos hidráulicos producidos a nivel nacional. Por ende, el presente 


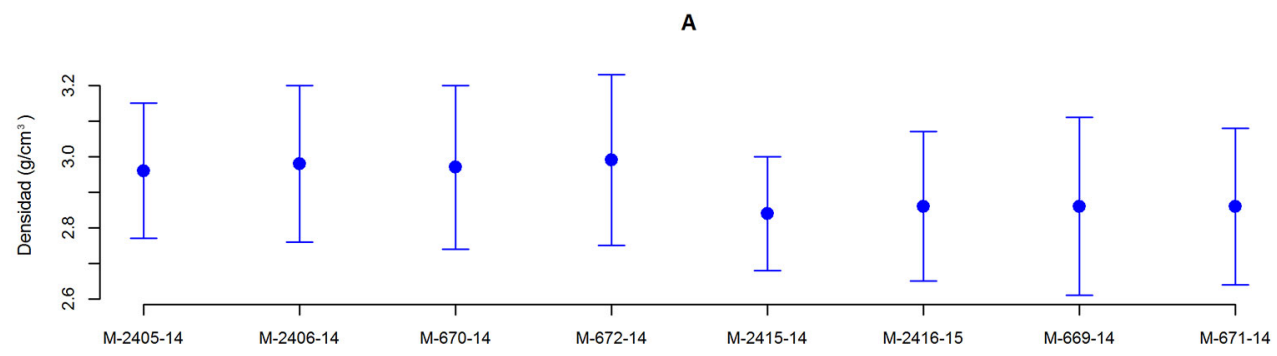

B

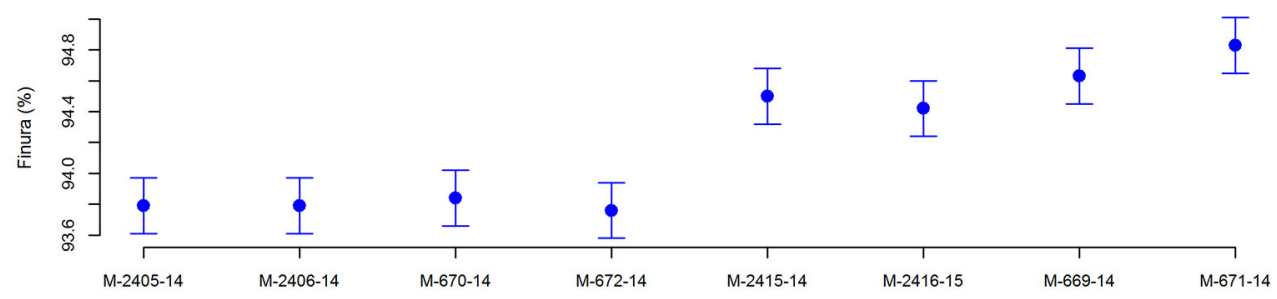

C

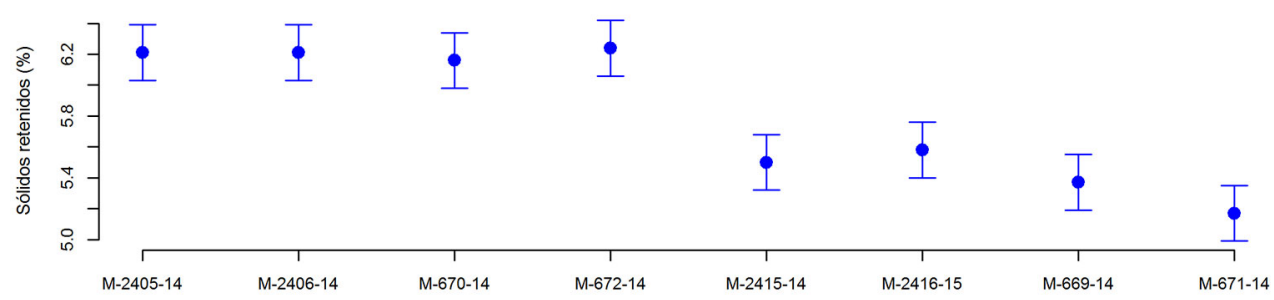

Figura 3. A) Densidades de los cementos hidráulicos de uso general muestreados, B) Finura obtenida en los cementos hidráulicos de uso general muestreados, C) Residuos sólidos obtenidos en el tamiz de $45 \mu \mathrm{m}$.

estudio detalla y brinda información relevante a la comunidad científica y público en general sobre los porcentajes promedio de los componentes mayoritarios y minoritarios en el cemento hidráulico de uso general que se comercializa en Costa Rica $(24,22)$.

En el tabla 2 se aprecia que la constitución química de los cementos nacionales tienen una alta similitud con los cementos alemanes identificados como: cemento Portland con resistencia a la compresión de 32,5 MP a los 28 días (CEM I - 32,5), cemento Portland con resistencia a la compresión de 42,5 MP a los 28 días (CEM I- 42,5), cemento Portland de alta resistencia con caliza (CEM II/A-LL), cemento Portland compuesto de baja resistencia con caliza y cenizas volantes silícea (CEM II/B-M (V-LL)) y cemento Portland de alta resistencia con escoria de alto horno (CEM II/A-S) (30). Sin embargo, se observa que todos los cementos comercializados en Costa Rica, al igual que los cementos alemanes anteriormente descritos, poseen como componente mayoritario el $\mathrm{CaO}$, el cual es adicionado principalmente por el clinker y la caliza. 
84 VENEGAS, CALDERÓN, SIBAJA Y OTROS: Contenido de metales pesados y composición química...

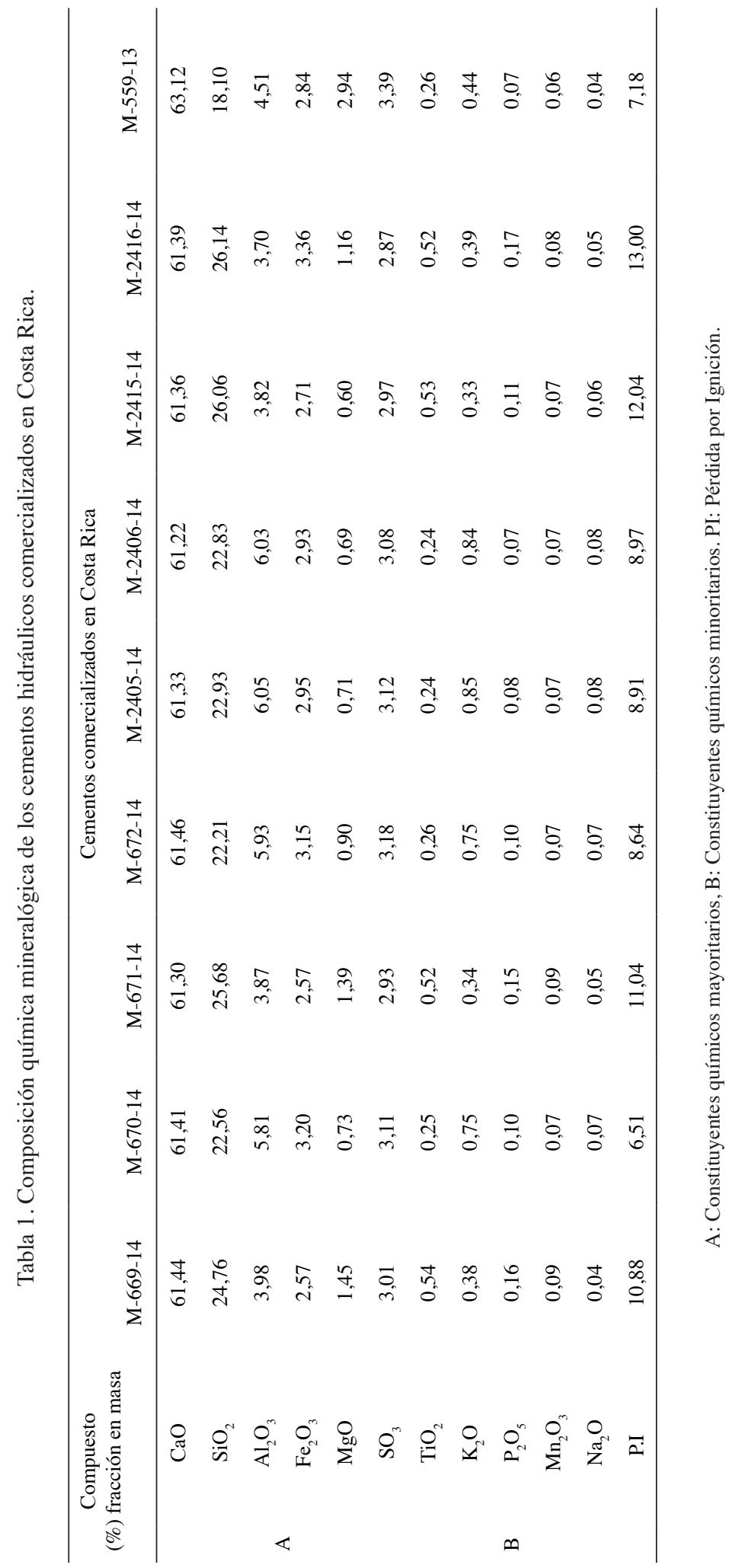




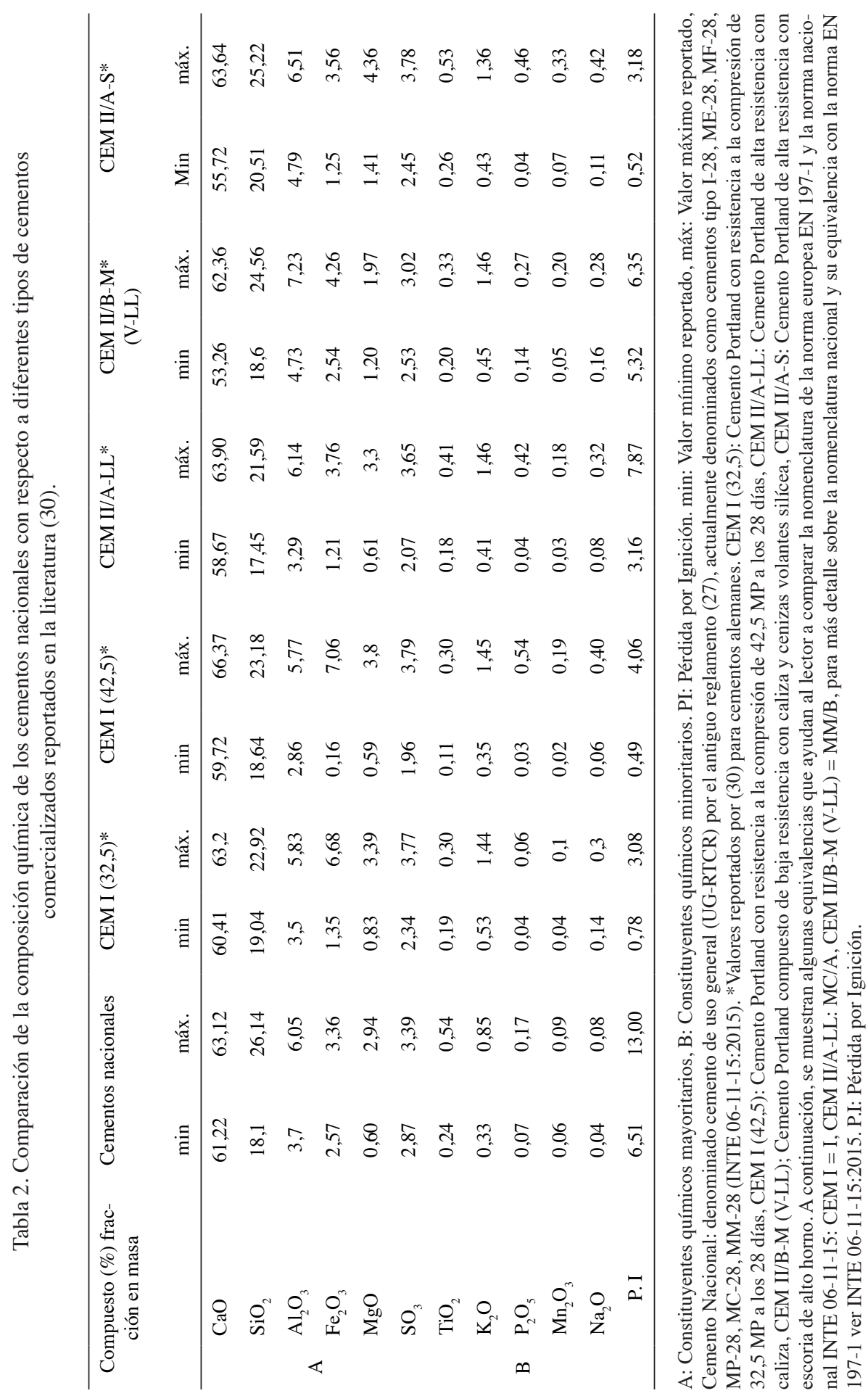


86 VENEGAS, CALDERÓN, SIBAJA Y OTROS: Contenido de metales pesados y composición química...

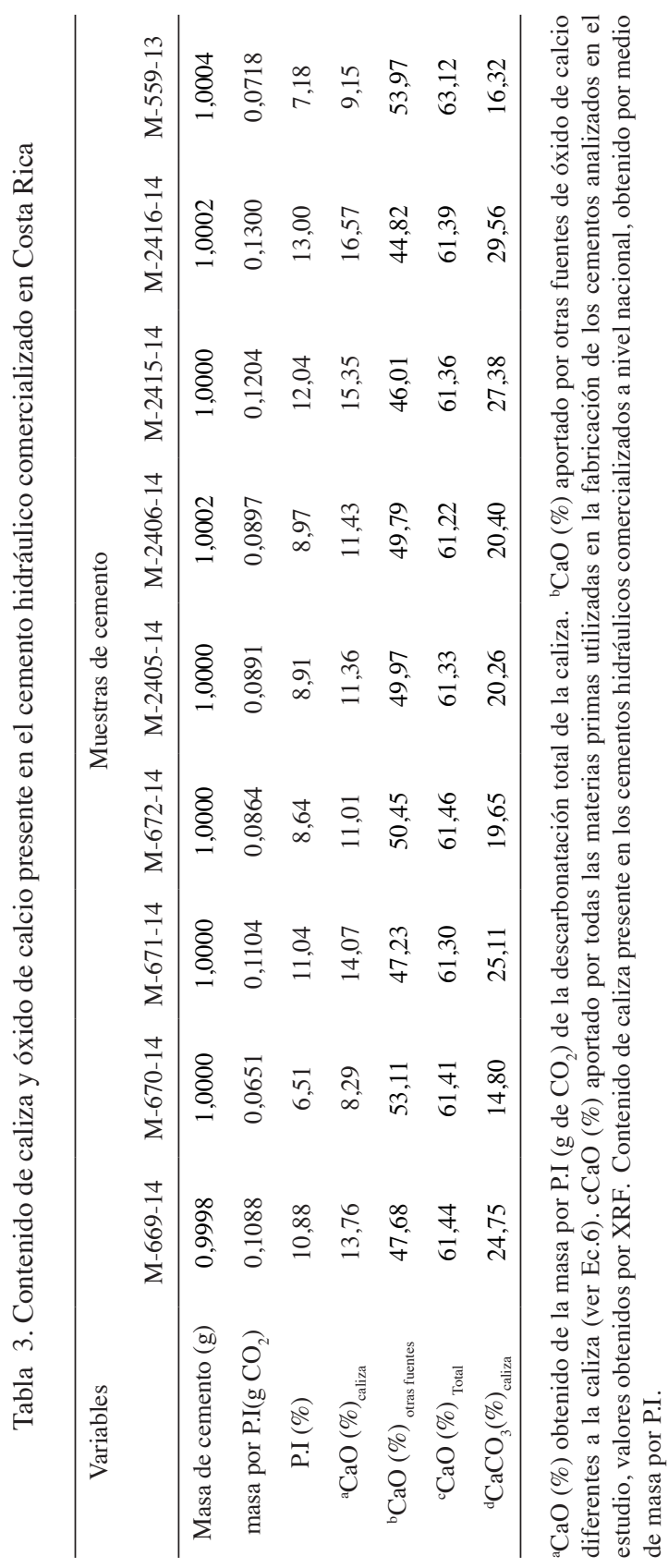


Al comparar el contenido de los compuestos minoritarios $\left(\mathrm{TiO}_{2}, \mathrm{~K}_{2} \mathrm{O}, \mathrm{P}_{2} \mathrm{O}_{5}, \mathrm{Mn}_{2} \mathrm{O}_{3}, \mathrm{Na}_{2} \mathrm{O}\right)$ presente en los cementos nacionales, se observa que, en la mayoría de los casos, el contenido mínimo y máximo obtenidos en este estudio son superiores a los reportados en los cementos alemanes (30). Lo anterior se asocia a las diferencias geoquímicas de las materias primas y de las características químicas de los materiales alternos utilizados, así como los diferentes porcentajes en que son introducidos estos materiales en los cementos alemanes y costarricenses.

En términos generales, se puede apreciar que la pérdida por ignición en los cementos nacionales es mayor a un $6,51 \%$, lo que indica que los cementos analizados contienen un porcentaje considerable de caliza en su formulación. Sin embargo, como se observa en la tabla 2, algunos cementos incorporan dentro de su constitución el uso de caliza como material suplementario, y presentan una gran similitud en los porcentajes de pérdida de ignición con los cementos nacionales; ejemplo de lo anterior son los cementos europeos tipo CEM II/A-LL y el CEM II/B-M (V-LL) (30).

En concordancia con lo anterior, a partir de los resultados de la prueba de ignición, se estimó el contenido de $\mathrm{CaO}$ aportado por la caliza en los cementos comercializados en Costa Rica (ver tabla 3). Lo anterior se realizó estableciendo una reacción completa de descarbonatación de la caliza y asumiendo que todo el carbonato presente en el cemento proviene del $\mathrm{CaCO}_{3}$, como se puede apreciar en la siguiente ecuación química:

$$
\mathrm{CaCO}_{3(s)}+\text { Energía } \rightarrow \mathrm{CaO}_{(s)}+\mathrm{CO}_{2}(g)
$$

Además, a partir del contenido de $\mathrm{CaO}$ aportado por la caliza se pudo diferenciar el contenido de $\mathrm{CaO}$ aportado por otras fuentes, tal y como se puede observar en la siguiente expresión:

$$
\mathrm{CaO}(\%)_{\text {otras fuentes }}=\mathrm{CaO}(\%)_{\text {total }}-\mathrm{CaO}(\%)_{\text {caliza }}
$$

Los resultados del tabla 3 muestran que el $\mathrm{CaO}$ presente en el cemento nacional proviene de la caliza en porcentajes de entre el $8,29 \%$ y el 16,57 \%, mientras que un porcentaje elevado entre un $44,82 \%$ hasta un $53,97 \%$ proviene de otras fuentes, principalmente los componentes utilizados para producir el cemento hidráulico (clinker de cemento Portland y/o escoria granulada de alto horno). Lo anterior concuerda con los intervalos de adición de caliza establecidos para el cemento hidráulico de uso general (UG-RTCR), los cuales regulaba el antiguo reglamento RTCR 383, mismo que estaba en vigencia en el momento en que se realizó este estudio (27).

Además, empleando los resultados del $\mathrm{CaCO}_{3}(\%)$ proveniente de la caliza (ver tabla 3) y utilizando la nomenclatura de la nueva norma nacional de cementos, los cementos analizados en este estudio se podrían designar como cemento hidráulico con caliza tipo $\mathrm{MC} / \mathrm{A}$ o tipo MC/B, con un contenido entre el (6 - 20) \% o (21 - 35) \% de sustitución, 
respectivamente. Inclusive, estos cementos podrían designarse como cemento hidráulico modificado mixto (MM/B), en el cual el contenido entre el (21 - 35) \% de sustitución predomina la caliza como componente adicionado (26).

En lo que concierne al contenido de los metales pesados en los cementos comercializados en Costa Rica, un estudio previo recopiló y analizó los resultados reportados por la industria productora del cemento a nivel nacional (46). Este estudio identificó algunos vacíos técnicos que impedían una evaluación y/o verificación objetiva del contenido de estos elementos en la matriz de cemento. Dentro de los hallazgos encontrados se destacan la poca comparabilidad de los resultados emitidos entre los laboratorios, la falta de declaración de los procedimientos de toma de la muestra y el plan de muestreo, la falta de declaración de las incertidumbres de medida y algunas limitaciones en términos de cuantificación; específicamente, se observó la dificultad por parte de algunos laboratorios de cuantificar el mercurio en concentraciones inferiores a los $0,5 \mathrm{mg} \mathrm{kg}^{-1}$ (46).

Debido a lo anterior, el presente estudio tuvo como objetivo establecer de manera expedita el contenido de los metales pesados, específicamente el contenido de plomo $(\mathrm{Pb})$, cromo $(\mathrm{Cr})$ y mercurio $(\mathrm{Hg})$. En la tabla 4 se muestra el contenido de los metales pesados, específicamente $\mathrm{Cr}, \mathrm{Pb}$ y $\mathrm{Hg}$, presentes en los cementos hidráulicos analizados. Para el caso del $\mathrm{Cr}$, este puede estar presente en el cemento con diferentes estados de oxidación; sin embargo, los más estables son el cromo trivalente $\left(\mathrm{Cr}^{3+}\right)$ y el cromo hexavalente $\left(\mathrm{Cr}^{6+}\right)$ (2). Su estado de oxidación es dependiente de la cantidad de oxígeno presente en el horno de calcinación, ya que las altas concentraciones de oxígeno favorecen la formación de los cromatos $\left(\mathrm{CrO}_{4}^{2-}\right)$, mientras que niveles bajos de oxígeno benefician la formación de los compuestos con $\mathrm{Cr}^{3+}(2)$. Por otra parte, a nivel toxicológico los compuestos de $\mathrm{Cr}^{6+}$ son más tóxicos que los de $\mathrm{Cr}^{3+}$, ya que los oxianiones de $\mathrm{Cr}^{6+}$, como los $\mathrm{CrO}_{4}^{2-}$, penetran la membrana celular mediante el sistema de transporte de sulfatos, mientras que el $\mathrm{Cr}^{3+}$ no puede ser absorbido por esta vía (10). Cuando el $\mathrm{CrO}_{4}^{2-}$ entra

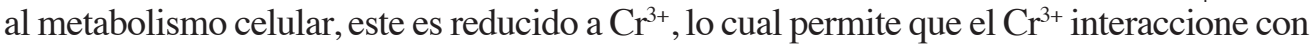
los ácidos nucleicos, las proteínas y los nucleótidos. Las interacciones del $\mathrm{Cr}^{3+}$ con las proteínas y el ADN aumentan la probabilidad de causar daños en el ADN, y por consiguiente la aparición del cáncer (10). Todo lo anterior, recalca la importancia de establecer el contenido de Cr presente en el cemento.

Como se observa en el tabla 4 , el $\mathrm{Cr}$ es el elemento que presenta mayor concentración en los cementos evaluados en este estudio. Este resultado es congruente con lo establecido para las diversas materias primas empleadas en la producción del cemento hidráulico en donde, dependiendo de la naturaleza mineralógica, la concentración de Cr puede encontrarse entre los $16 \mathrm{mg} \mathrm{kg}^{-1}$ (en piedra caliza) hasta los $100 \mathrm{mg} \mathrm{kg}^{-1}$ (en materias primas como la arcilla y las rocas margas) (2). Por otra parte, los combustibles alternativos como el carbón y el aceite, pueden contener un $80 \mathrm{mg} \mathrm{kg}^{-1}$ y un 50 $\mathrm{mg} \mathrm{kg}^{-1}$ de $\mathrm{Cr}$, respectivamente; por lo tanto, las fuentes naturales y antropogénicas podrían ser una explicación razonable de las concentraciones del $\mathrm{Cr}$ encontradas. De manera alterna, el Cr puede también ser introducido a la matriz del cemento por medio de la adición de la bauxita (material auxiliar). Aproximadamente, la bauxita se encuentra en el cemento en concentraciones del $4 \% \mathrm{~m} / \mathrm{m}$, y típicamente contiene concentraciones de entre $0,04 \% \mathrm{~m} / \mathrm{m}$ y $4 \% \mathrm{~m} / \mathrm{m}$ de $\mathrm{Cr}_{2} \mathrm{O}_{3}$ (2). Por último, los revestimientos 
refractarios en el horno de calcinación son otra posible fuente de $\mathrm{Cr}$ en el cemento (2, 9). Es importante destacar que pese a encontrarse un contenido significativo de $\mathrm{Cr}$ en los cementos analizados, este está dentro de los valores convencionalmente establecidos para la matriz de cemento (ver tabla 5).

Tabla 4. Concentración promedio de $\mathrm{Cr}, \mathrm{Pb}$ y $\mathrm{Hg}$ con su respectiva incertidumbre expandida, obtenida en los cementos muestreados

\begin{tabular}{lccc}
\hline Muestra & Concentración promedio con su respectiva incertidumbre expandida con un $k=2$ & $\begin{array}{c}\mathrm{Cr} \\
\left(\mathrm{mg} \mathrm{kg}^{-1}\right)\end{array}$ & $\begin{array}{c}\mathrm{Hg} \\
\left(\mathrm{mg} \mathrm{kg}^{-1}\right)\end{array}$ \\
\hline M-669-14 & $12,06 \pm 0,63$ & $3,33 \pm 0,40^{\mathrm{b}}$ & $0,136 \pm 0,024$ \\
M-670-14 & $23,33 \pm 0,76$ & $8,54 \pm 1,15^{\mathrm{a}}$ & $0,141 \pm 0,021$ \\
M-671-14 & $16,22 \pm 1,71$ & $2,45 \pm 0,72^{\mathrm{b}}$ & $0,084 \pm 0,012$ \\
M-672-14 & $22,03 \pm 1,65$ & $4,09 \pm 0,49^{\mathrm{a}}$ & $0,115 \pm 0,015$ \\
M-2405-14 & $23,86 \pm 1,19$ & $8,95 \pm 1,34^{\mathrm{a}}$ & $0,077 \pm 0,009$ \\
M-2406-14 & $21,62 \pm 1,18$ & $3,89 \pm 0,85^{\mathrm{b}}$ & $0,052 \pm 0,007$ \\
M-2415-14 & $15,31 \pm 0,94$ & $2,66 \pm 0,70^{\mathrm{b}}$ & $0,064 \pm 0,006$ \\
M-2416-14 & $10,69 \pm 0,92$ & $3,16 \pm 0,65^{\mathrm{b}}$ & $<\mathrm{LOQ}$ \\
\hline
\end{tabular}

${ }^{\mathrm{a}}$ Determinado por la técnica de FAAS, ${ }^{\mathrm{b}}$ Determinado por la técnica de GFASS, ${ }^{\mathrm{c}}$ Determinado por la técnica de CVAAS. $\mathrm{LOQ}=0,43 \mu \mathrm{g} \mathrm{kg}-1$

Tabla 5. Comparación del contenido de $\mathrm{Cr}, \mathrm{Pb}$ y $\mathrm{Hg}$ en algunas materias primas y en algunos cementos reportados en la literatura $(12,42,43)$

\begin{tabular}{cccccccccccc}
\hline Elemento & $\begin{array}{c}\text { Contenido }\left(\mathrm{mg} \mathrm{kg}^{-1}\right) \\
\text { cemento nacional }\end{array}$ & \multicolumn{1}{c}{$\begin{array}{c}\text { Contenido }\left(\mathrm{mg} \mathrm{kg}^{-1}\right) \\
\text { reportado en materias primas }\end{array}$} & \multicolumn{3}{c}{$\begin{array}{c}\text { Contenido }\left(\mathrm{mg} \mathrm{kg}^{-1}\right) \\
\text { reportado en diversos tipos de } \\
\text { cemento }\end{array}$} \\
\cline { 2 - 13 } & Mínimo & Máximo & $\mathrm{A}$ & $\mathrm{B}$ & $\mathrm{C}$ & $\mathrm{D}$ & $\mathrm{E}$ & $\mathrm{F}$ & $\mathrm{G}$ & $\mathrm{H}$ \\
\hline $\mathrm{Cr}$ & $10,69 \pm 0,92$ & $23,86 \pm 1,19$ & 14 & 66 & 25 & 172 & 41 & 40 & 68 & 50 \\
$\mathrm{~Pb}$ & $2,45 \pm 0,72$ & $8,95 \pm 1,34$ & 18 & 24 & 6 & 257 & 14 & 27 & 27 & 13 \\
$\mathrm{Hg}$ & $<$ LOQ & $0,141 \pm 0,021$ & 0,04 & 0,1 & 0,6 & 0,3 & 0,06 & 0,07 & 0,3 & 0,1 \\
\hline
\end{tabular}

A: Caliza (12); B: Clinker (12), C: Escoria de alto horno (12), D: Cenizas volantes (12), E: Cemento estándar (42), F: Cemento estándar (43), G: Cemento Portland (12), H: Cemento Modificado con Escoria de Alto Horno (12).

Por otra parte, el valor refleja el contenido total del Cr, el cual es la combinación del $\mathrm{Cr}^{3+} \mathrm{y} \mathrm{Cr}^{6+}$. Actualmente, a nivel nacional e internacional, existe la legislación y la normativa que protege a los trabajadores de la industria del cemento ante la exposición al $\mathrm{Cr}^{6+}$, por sus efectos dermatológicos, alergénicos y su evidencia carcinogénica $(8$, 12). Sin embargo, poco se conoce sobre el contenido de $\mathrm{Cr}^{6+}$ presente en los cementos comercializados en Costa Rica.

En el caso del $\mathrm{Pb}$, este elemento puede ser introducido al cemento por la materia prima, esencialmente por la arcilla y la pizarra $(2,12)$. Otras materias primas como la 
caliza, el clinker y la escoria de alto horno y cenizas volantes, también contribuyen al contenido del $\mathrm{Pb}$ en la matriz de cemento, siendo las cenizas volantes las que más contienen metales pesados en términos generales (ver tabla 5). Estudios demuestran que la concentración promedio del $\mathrm{Pb}$ en el cemento tipo portland es en promedio de $27 \mathrm{mg}$ $\mathrm{kg}^{-1}$, mientras que para los cementos puzolánicos con alto contenido de materiales silíceos y alumino-silíceos, la concentración de $\mathrm{Pb}$ puede disminuir hasta un promedio de $13 \mathrm{mg} \mathrm{kg}^{-1}$, dependiendo del tipo de puzolana con que se fabrica el cemento (ver tabla 5). Además, los combustibles alternativos son otra fuente de incorporación del $\mathrm{Pb}$, ya que está presente en altas concentraciones en los aceites lubricantes usados, en los neumáticos desechados y en el carbón. Los efectos del $\mathrm{Pb}$ en las propiedades mecánicas y químicas del cemento no se conocen con detalle; algunas investigaciones señalan que retarda principalmente la hidratación de las pastas (2).

La importancia de la determinación y la cuantificación del $\mathrm{Pb}$ en el cemento radica en el alto grado de toxicidad que posee este elemento. La toxicidad del $\mathrm{Pb}$ depende del tipo de especie y de la solubilidad de sus sales; por ejemplo, la especie más tóxica es el $\mathrm{Pb}$ iónico $\left(\mathrm{Pb}^{2+}\right)$. Los cloruros de $\mathrm{Pb}$ son ligeramente solubles en agua, mientras que los óxidos y los sulfatos de $\mathrm{Pb}$ son menos solubles en agua (10). En los microorganismos, el $\mathrm{Pb}$ puede ser bioacumulado, además retarda la descomposición de la materia orgánica por medio de los heterótrofos. Las investigaciones con los animales han indicado que el $\mathrm{Pb}$ es capaz de generar tumores (37). Además, el $\mathrm{Pb}^{2+}$ interacciona fuertemente con los grupos sulfhídrilos de las proteínas, ocasionando lesiones importantes en muchos de los órganos y afectando el sistema nervioso central y periférico, al inducir efectos secundarios encefalopáticos y de comportamiento, y además, afectando el funcionamiento de los riñones $(11,13,14)$. En el presente estudio se ha demostrado que los cementos muestreados presentaron una concentración de $\mathrm{Pb}$ entre los $(2,45 \pm 0,72) \mathrm{mg} \mathrm{kg}^{-1}$ y los $(8,95 \pm 1,34) \mathrm{mg} \mathrm{kg}^{-1}$ (ver tabla 4). Al comparar estas concentraciones con lo establecido en el antiguo reglamento de cemento hidráulico (38) solamente uno de los cementos muestreados presentó concentraciones superiores al límite indicado. Sin embargo, al igual que lo observado con el $\mathrm{Cr}$, el contenido de $\mathrm{Pb}$ determinado en los cementos nacionales analizados en este estudio, se encontró dentro de los valores convencionales establecidos por la literatura para la matriz de cemento (ver tabla 5).

En lo que concierne al $\mathrm{Hg}$, es un metal que, hasta el momento, no posee y/o no se conoce un efecto beneficioso o nutricional para al ser humano, siendo catalogado como extremadamente tóxico (13). Los compuestos de $\mathrm{Hg}$ menos tóxicos son los compuestos inorgánicos, debido a que no se absorben fácilmente en el tracto gastrointestinal por su baja solubilidad lipídica, pero todas las formas de $\mathrm{Hg}$ son tóxicas en un grado variable $(13,15)$. Específicamente en el cemento, este metal pesado puede ser incorporado en el proceso de clinkerización, por medio de los combustibles o de las materias primas que contengan trazas de $\mathrm{Hg}$ (ver tabla 5); por tanto, la mayor cantidad de este elemento se va a encontrar en el cemento en su forma elemental $\left(\mathrm{Hg}^{0}\right)$ u oxidada $\left(\mathrm{Hg}^{1+} \mathrm{y} \mathrm{Hg}^{2+}\right)$. $\mathrm{La}$ concentración del Hg también va a depender de la temperatura del proceso de calcinación. A temperaturas muy altas del horno, las cuales son usuales en los procesos de clinkerización, el Hg se volatiza y es emitido ya sea en los gases de combustión 
del horno o incorporado en el polvo de horno (CKD, por sus siglas en inglés) recolectado por los ciclones o por los precipitadores electrostáticos $(2,31)$. Esto provoca que en muchas ocasiones la concentración del mercurio sea muy baja. Sin embargo, en otras ocasiones, dependiendo del tipo de práctica industrial, el CKD puede ser introducido nuevamente al proceso de producción del cemento (45), lo que puede generar que la concentración de los elementos volátiles como el $\mathrm{Hg}$ sean incorporados nuevamente a la matriz de cemento (12). Lo descrito anteriormente, concuerda con los niveles encontrados del $\mathrm{Hg}$ en las muestras del cemento hidráulico analizadas, los cuales se distribuyeron entre los $52 \mu \mathrm{g} \mathrm{kg}^{-1}$ hasta los $141 \mu \mathrm{g} \mathrm{kg}^{-1}$ (ver tabla 4). Como se puede observar en términos comparativos, los cementos hidráulicos nacionales poseen en algunos casos un mayor contenido de $\mathrm{Hg}$ que lo reportado para diversos tipos de cementos europeos producidos en Alemania (ver tabla 5). Sin embargo, en algunas ocasiones, se observó que la concentración de este elemento fue tan baja que no se pudo detectar mediante la técnica de CVAAS (ver muestra M-2416-14, tabla 4). Una alternativa para mitigar este inconveniente analítico es utilizar técnicas de medición con mejores capacidades; específicamente, las técnicas, como por ejemplo, del Plasma de Acoplamiento Inductivo acoplado a detectores de Masas (ICP-MS) o la Dilución Isotópica con Plasma de Acoplamiento Inductivo Acoplado a Detectores de Masas (ID- ICP-MS), entre otras.

Finalmente, es importante recalcar que el creciente uso de los hornos cementeros como sistemas de co-procesamiento y de tratamiento de los residuos podría aumentar la concentración de estos elementos en la matriz del cemento. Debido a lo anterior, el presente estudio brinda los esfuerzos del sector académico y gubernamental para establecer y evaluar el contenido de los metales pesados $\mathrm{Cr}, \mathrm{Pb}$ y $\mathrm{Hg}$ en los cementos comercializados en Costa Rica, generando un aporte a la base científica para futuras investigaciones en el área reglamentaria, normativa y toxicológica.

\section{CONCLUSIONES}

El presente estudio demostró que los cementos hidráulicos distribuidos en Costa Rica presentan una constitución mineralógica adecuada, cumpliendo con estándares internacionales y nacionales de calidad. Además, análisis indirectos como los planteados en este estudio podrían ser utilizados para corroborar el uso de otros materiales alternos en la formulación de los cementos de uso general distribuidos en Costa Rica (tales como la caliza), que son comúnmente consumidos en la industria del cemento para mejorar las propiedades mecánicas y físicas de los mismos. Asimismo, se observó que el contenido de $\mathrm{Pb}$ y $\mathrm{Cr}$ en los cementos nacionales presenta menor concentración que el encontrado en otros tipos de cementos. Pese a lo anterior, es importante mencionar que el Cr fue el metal pesado que presentó mayor concentración en la matriz del cemento, específicamente en los cementos hidráulicos de uso general; sin embargo, el resultado es congruente con la naturaleza mineralógica de las materias primas que típicamente son utilizadas en la fabricación del cemento y presentan cantidades apreciables de $\mathrm{Cr}_{2} \mathrm{O}_{3}$. Aunque el contenido de $\mathrm{Cr}$ se encuentra dentro de las concentraciones reportadas por algunos estudios $(12,35)$ para este tipo de matriz, el resultado obtenido 
en este estudio no permite diferenciar entre las distintas especies $\left(\mathrm{Cr}^{3+}\right.$ y $\left.\mathrm{Cr}^{6+}\right)$. Por lo tanto, es necesario apuntar las futuras investigaciones a establecer el contenido de las diferentes especies del $\mathrm{Cr}$ presente en el cemento; específicamente, hay que realizar un gran esfuerzo para caracterizar y estudiar el contenido de $\mathrm{Cr}^{6+}$ en los cementos comercializados en Costa Rica.

Por otra parte, las concentraciones del Hg encontradas en algunas de las muestras analizadas en este estudio, son en comparación mayores a los reportados por la literatura $(12,42,43)$. Una posible explicación a esto puede ser la utilización de alguna materia prima o combustible alternativo que introduzca a la constitución del cemento un mayor contenido de $\mathrm{Hg}$. Sin embargo, para establecer estrictamente lo anterior, es necesario realizar un estudio más profundo en donde se evalúen cada una de las materias primas y combustibles alternos utilizados en la formulación de los cementos hidráulicos nacionales.

Cabe destacar que este estudio solo se enfocó en los elementos $\mathrm{Pb}, \mathrm{Cr}$ y $\mathrm{Hg}$ debido a su alto potencial toxicológico. Sin embargo, hay otros metales que deben ser estudiados en este tipo de cemento y otros tipos de cemento que se comercializan en Costa Rica, como por ejemplo (Cd, Hg, Pb, Ag, Cr, Ba, Ta, Sb, Ni, Be, Ag y Sr). Se espera que los resultados aquí demostrados, sobre los ámbitos de concentración en que se distribuyen estos elementos en el cemento, sirvan como base científica en la realización de estudios toxicológicos, estudios ingenieriles tales como los estudios de lixiviación, estabilización y encapsulación de los metales pesados en las matrices del concreto y sus agregados, con la finalidad de entender y establecer cómo estos metales se difunden desde la matriz de concreto hacia el ambiente y sus alrededores. Además, se espera que fortalezca los procesos de medición en este tipo de matriz y sirva de base para procesos de medición, monitoreo, normalización y reglamentación técnica nacional.

\section{AGRADECIMIENTOS}

Se agradece al Laboratorio Nacional de Materiales y Modelos Esturcturales (LANAMME) y al Laboratorio Costarricense de Metrología (LACOMET) por financiar y proveer los insumos necesarios para la realizar este estudio. Especialmente los autores desean agradecer a Guillermo González Beltrán, Coordinador del Laboratorio de Materiales, por su constante apoyo en este proyecto. El proyecto contó con financiamiento del LANAMME y con fondos de partida nacional del Ministerio de Economía, Industria y Comercio (MEIC) concedidos al LACOMET para desarrollar investigaciones para mejorar el Sistema Nacional para la Calidad de Costa Rica. Se agradece a la industria del cemento por brindar asistencia en el uso del RXF.

\section{REFERENCIAS}

1. Schönsleben P, Vodicka M, Bunse k, Ernst F.O. The changing concept of sustainability and economic opportunities for energy-intensive industries. CIRP Annals - Manuf Technol. 2010; 59 (1): 477-480. 
2. Bhatty J. Role of Minor Elements in Cement Manufacture and Use. Res Dev. 1995 Disponible en: http://www2.cement.org/DVD021.02/home.html

3. Lamas W, Fortes J. C, Camargo J. R. Waste materials co-processing in cement industry: Ecological efficiency of waste reuse. Renew Sustainable Energ Rev. 2013 March;19:202 - 3.

4. Edwin R, Dunstan Jr. Ash Library University of Kentucky, Denver: World of Coal Ash; 2011. Disponible en: http://energy.caer.uky.edu/AshSymposium/AshLibraryAgenda.asp\#2011

5. Schöler A, Lothenbach B, Winnefeld F, Zajac Maciej. Hydration of quaternary Portland cement blends containing blast-furnace slag, siliceous fly ash and limestone powder. Cement Concrete Comp. 2014;55(1):374.

6. Swiss Agency for the Environment, Forests and Landscape (SAEFL), Federal Office for the Environment (FOEN) Publications of the FOEN; 1998. Disponible en:

http://www.bafu.admin.ch/publikationen/publikation/00444/index.html?lang=en

7. Achternbosch M, Bräutigam K.R, Hartlieb N., Kupsch C, Richers U, \& Stemmermann, P. Impact of the use of waste on trace element concentrations in cement and concrete. Waste management \& research. 2005;23(4):328-37.

8. Chen, Q. Y., Tyrer, M., Hills, C. D., Yang, X. M., \& Carey, P. Immobilisation of heavy metal in cement-based solidification/stabilisation: a review. Waste Management. 2009;29(1):390-403.

9. Zhang J, Liu J, Li C, Nie Y, \& Jin Y. Comparison of the fixation of heavy metals in raw material, clinker and mortar using a BCR sequential extraction procedure and NEN7341 test. Cement and concrete research. (2008);38(5):675-80.

10. Wetterhahn J. K. The Role of Metals in Carcinogenesis: Biochemistry and Metabolism. Environ Health Persp. 1981 Aug;40(1):241.

11. Khan R.R. Environment and Metal Pollution, India: ABD Publishers: 2010.

12. Achternbosch M, Bräutigam K.R, Hartlieb N, Kupsch Chr, Richers U, Stemmermann P, et al., Karlsruher Institut für Technologie: ITAS - Publikationen 2003. Karlsruhe: Research Center of Karlsruhe $\mathrm{GmbH}$; 2003. Disponible en: http://www.itas.kit.edu/pub/l/j/lit03.htm

13. Goyer R, Golub M, Choudhury H, Hughes M., Kenyon E., Stifelman M. United States Environmental Protection Agency: Programs of the Office of the Science Advisor (OSA). Washington: ERG; 2004. Disponible en: https://www.epa.gov/osa/issue-paper-human-health-effects-metals 14. Needleman H. Lead Poisoning. Annu Rev Med. 2004;55(1):209-22. Disponible en: http://www.annualreviews.org/doi/abs/10.1146/annurev.med.55.091902.103653

15. European Commission: Scientific Committee on Toxicity, Ecotoxicity and the Envioronment (CSTEE). European Commissions Public Health: Publications. Brussels: CSTEE; 2002. Disponible en: http://ec.europa.eu/health/publications/index_en.htm

16. AEN/CTN 80 - Cementos y Cales: UNE-EN 196-7, AENOR: Buscador de normas. Madrid: AENOR; 2007. Disponible en: http://www.aenor.es/aenor/normas/buscadornormas/buscadornormas.asp?modob=S\#.VwlW-PnhDIU

17. American Society of Testing Materials (ASTM), ASTM International. West Conshohocken: ASTM International; ASTM C188 - 1995. Disponible en: http://www.astm.org/

18. American Society of Testing Materials (ASTM), ASTM International. West Conshohocken: ASTM International; ASTM C430 - 1996. Disponible en: http://www.astm.org/

19. Gebhardt F. R., editor. Rapid Methods for Chemical Analysis of Hydraulic Cement: STP 985. West Conshohocken: ASTM International; 1988. Disponible en: http://www.astm.org/ DIGITAL_LIBRARY/STP/stptocall.htm 
20. Calderón, B., Venegas, J., Salazar, J., Rodriguez, E. Determinación del contenido de plomo, cromo y mercurio en matriz de cemento mediante las técnicas analíticas de FAAS, GFAAS y CVAAS: Validación del método de ensayo. Revista Métodos y Materiales. 2016. En revisión e impresión

21. Bruce A.P. CEM Pioner und Marktführer der Mikrowellen-Labortechnik. Ohio: Systech Environmental Corporation. Disponible en: http://www.cem.de/

22. García R. H. Holcim España: Cementos Holcim Supercem. Valencia: Holcim; 2013 May 15. Disponible en: http://www.holcim.es/productos-y-servicios/cemento/productos/holcim-supercemr.html

23. Opoczky L, Gavel V. Effect of certain trace elements on the grindability of cement clinkers in the connection with the use of wastes. Int J Miner Process. 2004 Dec;74:S29-S30.

24. Narmluk M, Nawa T. Effect of fly ash on the kinetics of Portland cement hydration at different curing temperatures, Cement Concrete Res. 2011;41(1):580-1.

25. Taylor H. F. W. Cement Chemistry. 2nd ed. Great Britain: Academic Press; 2004.

26. CTN 06/ SC 10: PN INTE 06-11-15, INTECO: Normas nacionales INTE. Costa Rica: INTECO; 2015. Disponible en: http://inteco.or.cr/esp/centro-documentacion/catalogo-de-normas/ normas-nacionales-integdfghdfsa

27. Ministerio de Economía, Industria y Comercio. Decreto N 32253-MEIC: RTCR 383: 2004 Cementos Hidraúlicos. Especificaciones. La Gaceta 49. 2005 Mar 10.

28. Deolalkar S. P, Handbook for Designing Cement Plants. India: BS Publications; 2009.

29. Comité técnico AEN/CTN 80 Cementos y Cales: UNE-EN 197-1, AENOR: Buscador de normas. Madrid: AENOR; 2007. Disponible en: http://www.aenor.es/aenor/normas/buscadornormas/buscadornormas.asp?modob=S\#.VwlW-PnhDIU

30. VDZ: VDZ Activity Reports 2009 - 2012. Duesseldorf: VDZ GmbH; 2012. Disponible en: https://www.vdz-online.de/en/publications/activity-reports/

31. Smith, R. G. Determination of mercury in environmental samples by isotope dilution/ICPMS. Analytical Chemistry. 1993; 65(18), 2485-2488.

32. Elkhadiry I, Diouri A, Boukhary A, Rogez J. Mater Construcc., 2005;55:41.

33. ASTM E11-16 Standard Specification for Woven Wire Test Sieve Cloth and Test Sieves, ASTM International, West Conshohocken, PA, 2016, https://doi.org/10.1520/E0011-16

34. Decreto Ejecutivo N N 39297-MEIC. RTCR 476: 2015. Materiales de la Construcción. Cementos Hidráulicos. Procedimiento de Evaluación de la Conformidad. Gaceta del 17 de noviembre del 2015.

35. Eštoková A, Palaščáková L, Singovszká E, Holub M. Analysis of the chromium concentrations in cement materials. Procedia Engineering. 2012;42:123-30.

36. Guo Q. Increases of lead and chromium in drinking water from using cement-mortar-lined pipes: initial modeling and assessment. Journal of hazardous materials. 1997;56(1):181-213.

37. Ferrer, A. "Intoxicación por metales.” Anales del sistema sanitario de Navarra. Vol. 26. G

38. Reglamento Técnico RTCR 383:2004. Cementos Hidráulicos. Especificaciones, publicado en La Gaceta No. 49 del 10 de marzo de 2005. Departamento de Salud, 2003.

39. Taylor, H. F. W. Cement Chemistry, 2nd edition, editorial Academic Press: Great Britain; pp 1, 60-61, 64-65.

40. ASTM E1621-13 Standard Guide for Elemental Analysis by Wavelength Dispersive X-Ray Fluorescence Spectrometry, ASTM International, West Conshohocken, PA, 2013. Disponible en: https://doi.org/10.1520/E1621 
41. ISO 29581-2:2010- Cement - Test methods - Part 2: Chemical analysis by X-ray fluorescence. Ginebra. Suiza.

42. VDZ. "Umweltdaten der deutschen Zementindustrie". First edition. 2000. VDZ Verein Deutscher Zementwerke e.V. Disponible en: http://www.vdz-online.de

43. VDZ. Trace Elements in German Standard Cements. 2001. VDZ Verein Deutscher Zementwerke e.V. Disponible en: http://www.vdz-online.de

44. Siddique, R. Utilization of cement kiln dust (CKD) in cement mortar and concrete - an overview. Resources, conservation and recycling. 2006;48(4):315-38.

45. Taubert, D. H. Beneficial uses of cement kiln dust. In Cement Industry Technical Conference Record, 2008 IEEE:210-28.

46. Comisión Interinstitucional de Cemento Hidráulico. Informe. Tabla Decreto No 32253 MEIC. RTCR 383:2004 Cementos Hidráulicos Especificaciones. MINAE, MEIC, ProGAI-UCR, LACOMET. San Jose, Costa Rica. 2012. 